\title{
A note on solitary travelling-wave solutions to the transformed reduced Ostrovsky equation
}

\author{
E.J. Parkes ${ }^{\text {a }}$

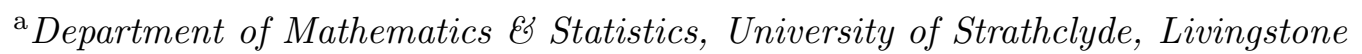 \\ Tower, Richmond Street, Glasgow G1 1XH, UK
}

\begin{abstract}
Two recent papers are considered in which solitary travelling-wave solutions to the transformed reduced Ostrovsky equation are presented. It is shown that these solutions are disguised versions of previously known solutions.
\end{abstract}

Key words: Solitary travelling-wave solutions; reduced Ostrovsky equation. PACS: 02.30.Jr.

Over the past two decades or so several methods for finding solitary travellingwave solutions to nonlinear evolution equations have been proposed, developed and extended. The solutions to dozens of equations have been found by one or other of these methods. References [1-3] and references therein mention some of this activity. Unfortunately, some authors claim that their solutions are 'new', when the truth is that these solutions are merely 'old' solutions in a different guise. Some authors give long lists of so-called 'new' solutions apparently unaware that some or all of the solutions are the same solution in disguise. Recently, in a series of enlightening papers [4-6], Kudryashov has warned researchers and referees of the danger of not recognizing that apparently different solutions may simply be different forms of the same solution. He has provided numerous examples to illustrate this phenomenon. Another recent example was given in [7]. In the present note we point out two more examples $[2,3]$ in which the equation under discussion is

$$
u u_{x x t}-u_{x} u_{x t}+u^{2} u_{t}=0 .
$$

This equation is a transformed form of the Vakhnenko equation [8] which, in turn, is a transformed version of the reduced Ostrovsky equation [9].

Solutions to (1) may be found by use of the tanh-function method. We have done this with minimal effort by use of the automated tanh-function method [10] which

Email address: e.j.parkes@strath.ac.uk (E.J. Parkes). 
uses ATFM, a Mathematica package designed to take the drudgery out of applying the tanh-function method by hand. The resulting solutions are

$$
u(x, t)=\frac{3 \alpha^{2}}{2} \operatorname{sech}^{2}\left[\frac{\alpha}{2}\left(x-\beta t-x_{0}\right)\right]
$$

and

$$
u(x, t)=-\alpha^{2}+\frac{3 \alpha^{2}}{2} \operatorname{sech}^{2}\left[\frac{\alpha}{2}\left(x-\beta t-x_{0}\right)\right],
$$

where $\alpha, \beta$ and $x_{0}$ are arbitrary constants. The solution (2) was given by the first equation in (3.7) in [8]. The solutions (2) and (3), with $x_{0}=0$, were derived by both the tanh-function method and by the 'rational function in exp' method in [1]. In [7] we explained why these two methods are entirely equivalent.

It is well known that for any (bounded) solution delivered by the tanh-method, there is a corresponding (unbounded) solution with tanh replaced by coth (see [11], for example). Hence

$$
u(x, t)=-\frac{3 \alpha^{2}}{2} \operatorname{cosech}^{2}\left[\frac{\alpha}{2}\left(x-\beta t-x_{0}\right)\right]
$$

and

$$
u(x, t)=-\alpha^{2}-\frac{3 \alpha^{2}}{2} \operatorname{cosech}^{2}\left[\frac{\alpha}{2}\left(x-\beta t-x_{0}\right)\right]
$$

are also solutions of (1).

In [2], the auxiliary-equation method was used to deliver 28 solutions, comprising four sets of seven. The authors claim to have found 'many new' solutions. First we observe that each set of seven can be written in the same way, namely

$$
\begin{aligned}
& u_{1}=\frac{3 \alpha^{2}}{2}\left(2 z_{1}-z_{1}^{2}\right), \quad \text { where } \quad z_{1}=\frac{\operatorname{sech}^{2} \eta}{1-\frac{1}{4}(1+\varepsilon \tanh \eta)^{2}}, \\
& u_{2}=-\frac{3 \alpha^{2}}{2}\left(2 z_{2}+z_{2}^{2}\right), \quad \text { where } \quad z_{2}=\frac{\operatorname{cosech}^{2} \eta}{1-\frac{1}{4}(1+\varepsilon \operatorname{coth} \eta)^{2}}, \\
& u_{3}=\frac{3 \alpha^{2}}{2}\left(2 z_{3}-z_{3}^{2}\right), \quad \text { where } \quad z_{3}=\frac{\operatorname{sech}^{2} \eta}{1-\varepsilon \tanh \eta}, \\
& u_{4}=-\frac{3 \alpha^{2}}{2}\left(2 z_{4}+z_{4}^{2}\right), \quad \text { where } \quad z_{4}=\frac{\operatorname{cosech}^{2} \eta}{1-\varepsilon \operatorname{coth} \eta} \\
& u_{5}=\frac{3 \alpha^{2}}{2}\left(2 z_{5}-z_{5}^{2}\right), \quad \text { where } \quad z_{5}=1+\varepsilon \tanh \eta, \\
& u_{6}=\frac{3 \alpha^{2}}{2}\left(2 z_{6}-z_{6}^{2}\right), \quad \text { where } \quad z_{6}=1+\varepsilon \operatorname{coth} \eta, \\
& u_{7}=\frac{4 a_{1} \alpha^{2} e^{2 \eta}}{\left(e^{2 \eta}+\frac{2 a_{1}}{3}\right)^{2}}
\end{aligned}
$$

where $\varepsilon= \pm 1, \eta=\alpha(x-\beta t) / 2$ and $a_{1}$ is an arbitrary constant. Each set of seven in [2] corresponds to a different expression for the arbitrary constant $\alpha$ in (6)-(12) 
above. For example, in the second set, $\alpha^{2} \equiv-a_{1}^{2} /\left(6 a_{2}\right)$, where $a_{2}<0$ is an arbitrary constant.

It is straightforward (but tedious) to verify that the expressions for $u_{1}$ and $u_{2}$ may be written as (2) and (4) respectively, with $x_{0}$ given by $\tanh \left(\alpha x_{0} / 2\right)=-\varepsilon / 3$. Also the expressions for $u_{3}$ and $u_{5}$ simplify to $(2)$ with $x_{0}=0$, and the expressions for $u_{4}$ and $u_{6}$ simplify to (4) with $x_{0}=0$. The expression for $u_{7}$ may be written in the form (2) with $x_{0}$ given by $\exp \left(\alpha x_{0}\right)=2 a_{1} / 3$. Consequently, all 28 solution expressions in [2] may be written in one or other of the forms (2) and (4). The authors of [2] cite [1] so must have been aware of the solution given by (2) with $x_{0}=0$.

In [3], application of the Exp-function method leads to two solutions that may be written

$$
u=\frac{3 \alpha^{2} b_{0}}{b_{1} e^{\xi}+b_{0}+\frac{b_{0}^{2}}{4 b_{1}} e^{-\xi}}
$$

and

$$
u=\frac{\alpha^{2}\left(-b_{1} e^{\xi}+2 b_{0}-\frac{b_{0}^{2}}{4 b_{1}} e^{-\xi}\right)}{b_{1} e^{\xi}+b_{0}+\frac{b_{0}^{2}}{4 b_{1}} e^{-\xi}},
$$

respectively, where $\xi=\alpha(x-\beta t)$, and $b_{0}$ and $b_{1}$ are arbitrary constants. In [3], it is claimed that (13) and (14) are 'novel' solutions. However, we observe that with $x_{0}$ defined by $\exp \left(-\alpha x_{0}\right)=2 b_{1} / b_{0},(13)$ and (14) may written in the form (2) and (3), respectively.

In [3], the authors go on to consider the special case with $b_{0}=2 b_{1}$ which corresponds to $x_{0}=0$; they simplify (13) and (14) to obtain

$$
u=\frac{3 \alpha^{2}}{1+\cosh [\alpha(x-\beta t)]}
$$

and

$$
u=-\alpha^{2}+\frac{3 \alpha^{2}}{1+\cosh [\alpha(x-\beta t)]},
$$

respectively. (The factor $3 \alpha^{2}$ was accidentally omitted in the latter solution; see (25) in [3].) Unfortunately the authors then failed to realize that the use of the identity

$$
1+\cosh 2 \theta=2 \cosh ^{2} \theta
$$

in (15) and (16) leads to (2) and (3) with $x_{0}=0$. However, since they cite [1], the authors must have been aware of the latter solutions!

\section{References}

[1] E. Yusufoğlu, A. Bekir, A travelling wave solution to the Ostrovsky equation, Appl. Math. Comput. 186 (2007), 256-260. 
[2] F. Kangalgil, F. Ayaz, New exact travelling wave solutions for the Ostrovsky equation, Phys. Lett. A 372 (2008) 1831-1835.

[3] C. Köroğlu, T. Öziş, A novel traveling wave solution for Ostrovsky equation using Exp-function method, Comput. Math. Appl. (2009), doi:10.1016/j.camwa.2009.03.028.

[4] N.A. Kudryashov, N.B. Loguinova, Be careful with the Exp-function method, Commun. Nonlinear Sci. Numer. Simulat. 14 (2009) 1881-1890.

[5] N.A. Kudryashov, On "new travelling wave solutions" of the KdV and KdV-Burgers equations, Commun. Nonlinear Sci. Numer. Simulat. 14 (2009) 1891-1900.

[6] N.A. Kudryashov, Seven common errors in finding exact solutions of nonlinear differential equations, Commun. Nonlinear Sci. Numer. Simulat. 14 (2009) 3507-3529.

[7] E.J. Parkes, A note on travelling-wave solutions to Lax's seventh-order KdV equation, Appl. Math. Comput. 215 (2009) 864-865.

[8] V.O. Vakhnenko, E.J. Parkes, The two loop soliton solution of the Vakhnenko equation, Nonlinearity 11 (1998) 1457-1464.

[9] E.J. Parkes, Explicit solutions of the reduced Ostrovsky equation, Chaos, Solitons \& Fractals 31 (2007) 602-610.

[10] E.J. Parkes, B.R. Duffy, An automated tanh-function method for finding solitary wave solutions to nonlinear evolution equations, Comput. Phys. Commun. 98 (1996) $288-300$.

[11] E. Fan, H. Zhang, A note on the homogeneous balance method, Phys. Lett. A 246 (1998) 403-406. 